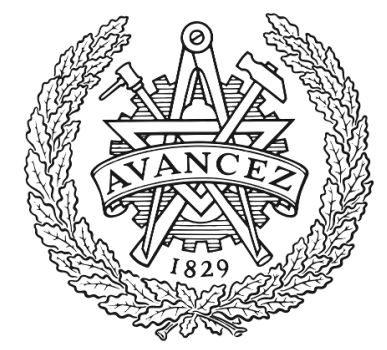

CHALMERS

UNIVERSITY OF TECHNOLOGY

\title{
Leverage Points for Focus Flow and Communitas
}

Downloaded from: https://research.chalmers.se, 2023-04-26 15:19 UTC

Citation for the original published paper (version of record):

Penzenstadler, B. (2020). Leverage Points for Focus Flow and Communitas. ACM International Conference Proceeding Series: 267-274. http://dx.doi.org/10.1145/3401335.3401826

N.B. When citing this work, cite the original published paper. 


\title{
Leverage Points for Focus Flow and Communitas
}

\author{
Birgit Penzenstadler \\ birgitp@chalmers.se \\ Chalmers|Gothenburg University \\ Göteborg, Sweden \\ Lappenranta Lahti University of Technology \\ Lappenranta, Finland
}

\begin{abstract}
Society is running at a high pace, and with it the hamster wheel we sometimes perceive ourselves to be in. In more scientific terms, this is called cognitive overload in combination with artificial deadline pressure. There is a notion of overwhelm in combination with perceived time scarcity in terms of the cognitive load of individuals. However, if we as individuals are not living in a sustainable way, how can we attempt to create a sustainable world?

This paper provides an autoethnography of the use of leverage points to reduce cognitive load for a computer worker, with insights from literature and self-experiments, as well as a discussion on what is needed for changes on a bigger scale. An understanding of how cognitive load and resilience can be addressed by choosing and using specific leverage points has the potential to increase individual sustainability and resilience, what can be called focus flow, as well as communitas (group flow).
\end{abstract}

\section{CCS CONCEPTS}

\section{- Social and professional topics; KEYWORDS}

individual sustainability, software engineering, resilience, cognitive load, leverage points

\section{ACM Reference Format:}

Birgit Penzenstadler. 2020. Leverage Points for Focus Flow and Communitas. In 7th International Conference on ICT for Sustainability (ICT4S2020), Fune, 2020, Bristol, United Kingdom. ACM, New York, NY, USA, 8 pages. https: //doi.org/10.1145/3401335.3401826

\section{INTRODUCTION}

For a large part of computer workers, which could be software engineers or academics but also many other jobs that require a computer for multiple hours per day, the permanent cognitive load they are under is too high, which increases stress and lowers productivity and creativity [7]. Some of the tendencies I observe are that there is an information flood to keep up with the (whichever way you choose to filter) most important world news, state of the art and practice in the field of our work, administrative processes at work and in societal community, etc. There is a movement from

Permission to make digital or hard copies of part or all of this work for personal or classroom use is granted without fee provided that copies are not made or distributed for profit or commercial advantage and that copies bear this notice and the full citation on the first page. Copyrights for third-party components of this work must be honored

For all other uses, contact the owner/author(s).

ICT4S2020, fune, 2020, Bristol, United Kingdom

(C) 2020 Copyright held by the owner/author(s)

ACM ISBN 978-1-4503-7595-5/20/06.

https://doi.org/10.1145/3401335.3401826 informing people to advertising, which added a pushing factor that spiraled out of control, and we even do it in academia now. For example, when we look at calls for participation that get pushed all over mailing lists and social media - and I have been part of the problem, having been publicity chair at too many conferences, trying to increase submission rates in an effort to create a good program.

We all have a specific baseline amount of energy each day (plus good or bad self-care habits that help keep that up or lower it, e.g. nutrition and exercise). Specifically, computer work is associated with high depression risk and anxiety, not only in self-reported studies [7] but also in cross-sectional studies that compared computer workers with manufacturing workers [6]. Symptoms of this input overload differ widely; we all have individual signs. For example, I experience dizziness from fuzzy thoughts spinning in my head, sometimes catch myself biting cuticles, get a buzzy high-pitched tone in my ear, or a twitching eyelid, or a cold sore. Another common symptom is overeating in a vain attempt for trying to get energy to fight against a sense of overwhelm caused by overflowing to-do lists, stimulation when the world feels dull (which is does because our sense shut down due to constant overstimulation), filling a perceived emptiness that is due to a lack of sense of purpose and willingness to look inside.

Research Gap: Pargman and Raghavan presented a comprehensive critical discussion of the buzzword-use of the term sustainability in computing and their proposal of non-negotiable limits [15], but one such non-negotiable limit that was not considered in the paper is the limit of how much work and online time is sustainable for humans and where that actually borderlines with their resiliency and healthy survival. I am interested in the transition towards focus flow and communitas (group flow [8]) - a feeling of connectedness with the Self, the work environment, and the world at large.

Research Objective: Discover what helps shift towards flow and communitas in a computer work dominated environment using the lens of leverage points.

Contribution: Interventions and self-experiments with the leverage points show that "Knowledge controls energy, and energy controls matter." While I can only contribute a limited autoethnography and discussion, the interventions have contributed to increased focus flow and communitas in my environment that may inspire others to replicate these efforts.

Impact: The wider application of this work could potentially lead to focus flow and communitas for individuals and teams who choose to try it. Resilience increases the capacity for compassion, 
which benefits the world at large. While I am definitely against measuring individual resilience in monetary amounts, I want to point out that this is beneficial for business, because resilient people are less likely to burn out, have fewer sick days, and produce higher quality work.

\section{BACKGROUND AND RELATED WORK}

The original Leverage Points paper by Donella Meadows describes places to intervene in a system from her vast experience in complex systems analysis [12]: Leverage points are, as Donella Meadows puts it, "an invitation to think more broadly about the many ways there might be to get systems to change". The trouble is, as she quotes Jay Forrester, that while "People know intuitively where leverage points are", "Everyone is trying very hard to push it in the wrong direction!" as he demonstrated to the Club of Rome for the major global problems (poverty and hunger, environmental destruction, resource depletion, urban deterioration, unemployment) with the leverage point of Growth [3]. Therefore, she set out to explain Leverage Points such that people would not only find them but also understand how they work in a system, such that they would try to push it in the direction that is actually helpful to the situation. Despite these efforts, she ends with pointing out the caveats: "The higher the leverage point, the more the system will resist changing it-that's why societies tend to rub out truly enlightened beings." [12]

Donella Meadows stated that "Leverage points are places within a complex system (a corporation, an economy, a living body, a city, an ecosystem) where a small shift in one thing can produce big changes in everything." Table 1 lists the leverage points (LPs) in increasing order of effectiveness according to Meadows. While all LPs can bring about change, the later ones are more likely to create significant changes to the system behavior but may also require more effort to implement. Meadows's LPs refer to any kind of change, whether enabled by software or not. In the main article, I use them as an analysis tool for exploring how software can trigger broader changes in societal systems. However, they are hard to identify and act on - they are not a silver bullet [17].

Leverage points for sustainability transformation by Abson et al. [1] proposes that a research agenda centred on the concept of deep leverage points can provide a coherent framework for such engagement with the root causes of unsustainability.

Flow. There is a significant amount of work on Flow [2] and Communitas [8], which are second theoretic foundation block for this work. Flow, also known colloquially as 'being in the zone', is the holistic sensation that people feel when they act with total involvement [2]. Communitas is defined as 'group flow', and Plato described ecstasis for individuals as well as groups as an altered state where our normal waking consciousness vanishes completely, replaced by an intense euphoria and a powerful connection to a greater intelligence [8].

Cognitive Load. On some of the interventions related to lowering cognitive load, Widdicks et al. investigated reducing online time [24, 25], Sengers analyzed overwork and how staying at a remote island taught here a slower pace of life [19], and Fullerton

\begin{tabular}{l|l} 
LP & Description \\
\hline 12 & $\begin{array}{l}\text { Constants, parameters, and numbers. Tweaking parameters } \\
\text { allows change to the intensity of the flows in systems but } \\
\text { rarely alters the underlying dynamics. }\end{array}$ \\
The sizes of buffers and other stabilizing stocks, relative \\
to their flows. Stabilize a system by adjusting the capacity \\
of its buffers, and make it more efficient by optimizing the \\
flow.
\end{tabular}

Table 1: Leverage points according to [12].

discussed the importance of designing for disconnecting [4]. I implemented several of the points in these publications in my personal practice and am including them in the leverage point examples.

In my own previous work, I have used the lens of leverage points to look at software engineering and how it can support our world in moving towards sustainability [17]. For ICT4S'20 I have proposed a research agenda on how to improve our Focus Flow (currently under review) [16]. 


\section{RESEARCH METHOD}

I used autoethnography and explorative analysis of leverage points in order to understand the system dynamics of cognitive load and how to change it. The subject of investigation was own daily routines, type of work tasks and input channels (writing time, service commitments, administration, email, state of the art and practice, news) to explore which interventions can lower the cognitive load. I performed a qualitative evaluation of their impact in reflective journaling (research diary) and over about ten years. The habits described in the entries are taken to provide the examples for the leverage point implementations. The analysis is focused on the exploration of the leverage points more than the specific data points of the journal as I consider the discussion of the implication of the leverage points and the opportunities they offer us more of a contribution to the research discourse than a detailed data analysis of my notes on personal habits. The discourse serves as basis for a wider discussion around the lens of leverage points.

\section{RESULTS: LEVERAGE POINTS TO REDUCE COGNITIVE LOAD AND INCREASE RESILIENCE}

The twelve leverage points are sorted in order of increasing effectiveness, meaning that the 12th leverage point (Parameters), is the easiest to change, but that change also has a smaller effect on the overall system, and the further down the list, the harder they are to change, e.g. the 5th (Rules of the system), but the more effective the change will be. For ease of use and conceptual understanding, they are divided into four groups: parameters, feedbacks, design, and intent.

In Tab. 1, I show an overview of exemplary leverage points in the area of cognitive load at a computer working space. The purposefully simplified scenario (see Fig. 2) includes work tasks such as creating digital artifacts, writing reports, corresponding per email, scheduling and attending meetings, being evaluated on performance, and contributing to management (which tends to repeat the same tasks on a different level of abstraction).

In the following, I describe 1) a reflection of the leverage point level in relation to academic work, 2) a reflection on what could be done to push that lever, 3) an example of an effort by me to change something to push that lever and the outcomes of that, and 4) a reflection on the impact on communitas.

LP 12, Constants, parameters, and numbers: Parameters are the least effective group of leverage points, but they are easy to get started with, and the fact that they are less effective than others does not mean there is not already a big difference to be noticed, depending on which parameter is chosen to be changed. The example parameter for the cognitive load scenario that was chosen is time that is spent on email per day. I have many colleagues who complain about this frequently, and unless you have a good tactic in place yourself, you may be too familiar with it. It is easy to spend undue amounts of time answering email, to the extent that it appears you are not getting any 'real' work done because so much time is spent communicating. ${ }^{1}$ While there are helpful rules to make email concise and to the point, I have also had colleague-friends wondering whether I was upset with them for answering short, so these rules apply to a certain extent. The most helpful parameter I have found is to only check emails twice a day and setting fixed time blocks for responding to emails. There are proponents of only answering emails if it is absolutely necessary and their inboxes are growing into scary dimensions, which I have found to be stressful and adding to my cognitive load, so I have discarded that strategy. While I do not always manage to check emails only twice daily and I sometimes do get stuck spending longer than my assigned time block for it, having the parameters set (and communicated in my footer) has proven to significantly reduce the perceived cognitive load caused by emails. I achieved an impact on communitas of this practice by sharing it in my email footer, which reads "Sane mail - checked twice daily :)" I do not know to how much change it has led, but this footer has been referred to by many individuals corresponding with me.

LP 11, Size of buffers: There is a widespread, bad habit of scheduling back-to-back meetings. I would rarely want to do that to someone else, but I used to do that to myself a lot. There are plenty of reasons for why that is not a good idea: I might actually want to gather my thoughts in between, review my notes, go to the bathroom, or refill my water bottle. On occasions, I have underestimated the time it takes me to get places, that a bus might be a few minutes late, or that the wind is against me while I'm pedaling frantically on my bicycle to make it on time. In addition, meetings sometimes run over, for lack of moderation, or for actually good and productive reasons, and if I schedule back-to-backs, they eat into the next meeting slot, perpetuating and worsening the phenomenon. The simplest most effective answer to this (outside of just not having meetings) is to plan buffer time and always have at least 15 minutes of unassigned time in between meetings. When I do manage to follow that rule, I experience significantly more satisfaction with the progress I am making over the day because I actually have a couple of minutes to reflect on the meeting, note down follow-up steps, and review my preparation for the next meeting as well as taking care of physical needs (stretching, bathroom, water). This improves communitas, as the energy I bring into a meeting affects the whole group, either positively or negatively.

LP 10, Structure of flow: In every organization I have ever worked at or worked with, there is a plethora of organizational processes and administrative procedures that have to be obeyed and adhered to. The documentation of these is often hard to find or not kept up to date. While simplifying the processes themselves is a higher leverage point ( 6 and 5), the structure of the flow can be smoothened by always making the next step clear. In the example of email communication, that could simply require a clear and concise statement at the end of the email what I am asking for and what is requested by when. Previously, and in many emails I have received, there was an extra loop for clarifying instructions and deadlines and other specifics. With a short list of questions that I have in my head and check before sending out an email - what am I asking for and by

\footnotetext{
${ }^{1}$ I acknowledge that not all email is created equal - there is important email communication, and there is also a tendency to overuse the medium or use it inadequately. It only serves as an example.
} 


\begin{tabular}{|l|l|l|l|}
\hline \multirow{5}{*}{ Parameters } & 12. Parameters & Time on email per day & Set fixed block, check twice daily \\
\cline { 2 - 4 } & 11. Size of buffers & Meeting times & 15 minutes between meetings \\
\cline { 2 - 4 } & 10. Structure of flow & Organizational processes & Simple instructions in emails \\
\hline \multirow{5}{*}{ Feedbacks } & 9. Length of delays & Email response time & Lengthening increased \\
\cline { 2 - 4 } & 8. Strength of neg loops & \# of commitments & Reducing the \# of commitments \\
\cline { 2 - 4 } & 7. Gain around pos loops & Where focus goes, energy flows & It's either 'Hell, yes!' or 'No.' \\
\hline \multirow{3}{*}{ Intent } & 6. Structure of info flows & Always online & Keep offline time blocks \\
\cline { 2 - 4 } & 5. Rules of the system & 'Bean counting' competition & Individual perception of impact \\
\cline { 2 - 4 } & 4. Power to change structure & Hierarchical, limited democracy & Change starts at individual level \\
\cline { 2 - 4 } & 3. Goals of the system & Competition over resources & Essentialism (focus on main thing) \\
\cline { 2 - 4 } & 2. Mindset / paradigm & Scarcity & Union / abundance \\
\cline { 2 - 4 } & $\begin{array}{l}\text { 1. Power to transcend } \\
\text { paradigms }\end{array}$ & Business as usual & $\begin{array}{l}\text { Conscious awareness / Samadhi / } \\
\text { It's all a game / unattached }\end{array}$ \\
\hline
\end{tabular}

Figure 1: Leverage points to reduce cognitive load and increase resilience in computer work

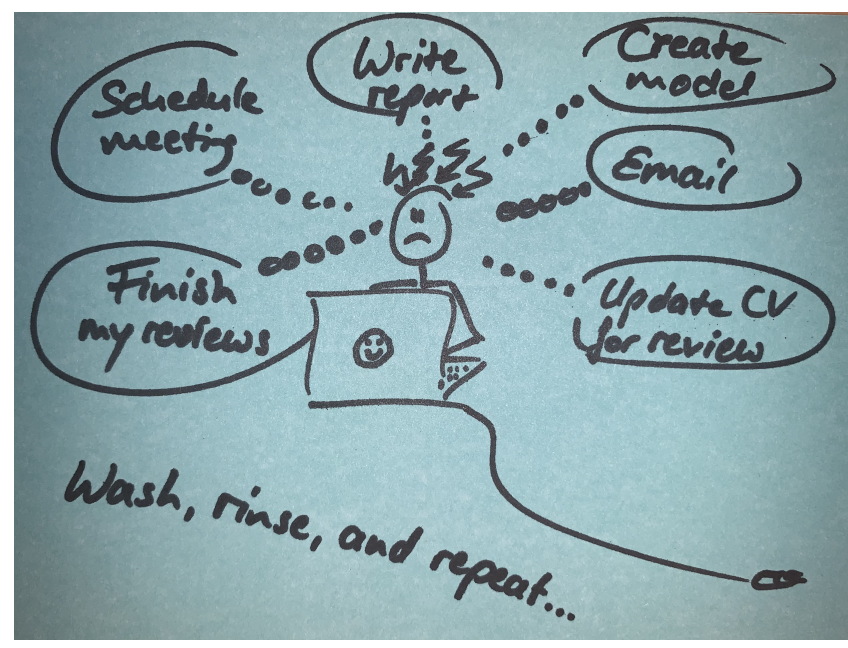

Figure 2: Scenario of cognitive load of computer work

when - I can avoid those loops. For me, this has proven beneficial in managing from the top (e.g. my students) as well as managing from below (e.g. my supervisors and team leaders) by timely and adequate responses to requests. Feedback shows that this increases communitas because it allows for more perceived individual agency.

LP 9, Length of delays: Email response time is a great example for us trying really hard to push the lever in the wrong direction. In the last decade, there has been a tendency to shorten email response times and that raises the expectations for always shorter response times. The result of it is an email load higher than ever and increased use of email as a quasi-synchronous communication medium whereas it was conceptualized as an electronic letter, and physical mail used to take between days and weeks to arrive. So when I experimented gently with pushing the lever the other way and waiting at least half a day to respond to (most, admittedly) emails, I observed a decrease in the frequency of quickly shot questions that did not require an email in the first place as well as more thoughtthrough replies and gentler pace in conversations. Here we perceive the effect on communitas as everyone in those conversations may note the decrease in perceived urgency as well.

LP 8, Strength of negative feedback loops: The number of commitments that we have can serve as an indicator for cognitive overload. If we have many commitments, we can spend only little time on each of them, which makes it more likely that we experience cognitive overload and actually get less done that we would otherwise, simply because switching contexts always requires additional cognitive resources and time, and because old commitments usually draw follow-ups after them as opposed to just fizzling out or being done. Consequently, the only way out of that negative feedback loop is to decrease its strength by reducing and strictly limiting our number of commitments. My personal cut-off for projects that I can really invest brains in over the course of one day is probably around three. That is rarely the number of things I have on my calendar - it is usually exceeded by double that at least. And while it is possible to get small steps done in many different projects if they are well defined, the ones that need some deep thinking and dedicated brain power need to get allocated in the best cognitive working time for the individual, for many of us in the morning. The positive effect on communitas is that I am contributing my best work to the groups I am part of by allocating my cognitive resources well.

LP 7 Gain around positive feedback loops: Where focus goes, energy flows. Knowledge or intention control energy, and energy controls matter. That means, the clearer I am on my intentions and why they matter, the easier it is to be motivated and, consequently, get things done. In my research work, that means when I have to decide whether to take on something or not, my ideal decision criterion is "It's either 'Hell, yeah!' or 'No.'” [21]. And you are right, this can be really hard to implement. However, when the priorities are clear, it is easier to take decisions. What has helped me in the last couple of years to make this criterion work is to remind myself that every time I say yes to a project proposal that I am not excited about, I am taking away from my time, so when an actual 'hell yeah' 
project were to come around the corner, I might not have enough time left to commit to that one. Every time I feel that I 'should', I take a minute to reflect on the occasions when I said yes because of a should, and most of those did not lead to glorious outcomes or memories. One area where this has to be taken with a grain of salt is personal relationships, because they always involve ups and downs, especially the romantic ones, but that is not the topic of this paper. In my work life, this has resulted in less overcommitment and in working on things I care about, one of which is the topic of this paper. In addition, every hour I invest into topics I really care about feels like it brings back more reward and deeper insights than when I work on something just to get it done. Where intention goes, energy flows. Same for communitas; if the group takes on too many commitments and spreads themselves too thin, we become ineffective and don't get things done. The 'we' I am talking about for this data point is the Karlskrona Alliance ${ }^{2}$, and every time we narrow down the agenda to a few items, we get incredibly effective.

LP 6 Structure of information flows: According to high performance coach Brendon Burchard, the worst thing we can do first thing in the morning is look into our emails. He describes your email inbox as a convenient organization of other people's to-do lists. The reason is that the emails you receive have requests that the senders want you to respond to. That may but more often may not align with how you prefer to choose your priorities for a given day. In addition, the constant interruption of an email client, if we are online all the time, makes productive working on other tasks close to impossible because of the decreased return on (time) investment the more often we have to switch context. Consequently, the reduction of online time and the adherence to offline time blocks allows for deep work time that is harder to get interrupted in. In my experiments over the years, trying to schedule writing blocks mid or late afternoon does not work very well, because sometimes things come in earlier during the day that need immediate attention and, in addition, I am just not at my cognitive best at that time anymore. So, my best practice is to schedule them first thing in the morning - and yes, it does take self-discipline to not start the email client until it has become a habit not to. I do my most productive writing offline, and it takes constant reminding myself of that fact in order to keep doing it.

LP 5 Rules of the system: Currently, most of our evaluation systems are based on some kind of competitive 'bean counting', with the intention to simplify things and to make them measurable. The reason is: What you cannot measure is much harder to control, and we humans seem to feel a strong need to be in control of things. Who sold the most products? Who published the most and most-cited papers? Can your evaluations compete with your colleagues'? Metrics make it possible to directly compare, but at the expense of relying on the meaningfulness of the metric instead of the actual work results of an individual. The consequence specifically in academia is that many early career researchers are worried about producing enough publications and how to slice the content such that the perceived outcome is the biggest. This has led to a significant inflation of many types of work results and to the undervaluation of any work that cannot contribute to the specific work metrics we get

\footnotetext{
${ }^{2} \mathrm{http}: / /$ www.sustainabilitydesign.org
}

measured by. There are counter-initiatives to help recognize other types of work, for example the peer review platform Publons to recognize reviewer efforts. That in itself deserves applause, and it is only the mitigation of the problem. Instead, there needs to be a shift towards an individual perception of impact. We are all part of a large organism, and the competitiveness is killing it - imagine an ant hill where the ants are competing; I doubt it would last long. I'm getting idealistic here, I know, but the joint vision is more important than counting who carried more bricks towards it. If researchers did not have to worry about whether their metrics are sufficient at the end of the day to keep their job (significant stress in terms of cognitive load), they might just produce even more meaningful results. I trust in my ability to survive enough to refuse to run after the metrics, and I have declined a number of invitations where all 'junior faculty symposiums' would say 'accept all those invites to establish yourself'. That may bite me later, and I am accepting of it, because I feel better aligned this way. That is not a big action nor a constructive proposal, I know, but not buying into the rules I disagree with is a first step to changing them. Surveys on the free salary for everyone have shown that the main concern people have is that others would slack off - no one stated they would not want it because they would not work themselves, so maybe our felt need to control is unjustified and only based on mistrust towards others? The rules of the system are a product of the community so for communitas, we need to come back to collaboration instead of competition, and it is on us to explore how to make that happen.

LP 4 Power to change structure: The structure our economy operates in, including our universities, is hierarchical with a limited democracy. In some countries and organizations there is more democracy, in others less, but all in all it is a combination of hierarchy and democratic influences. A lot of the hierarchy is dominated by money and conversations around money. Yes, most of us do need financial means to survive and to take care of ourselves and others, but if it dictates the conversation, we keep spiraling towards the elusive growth that Meadows already showed in the seventies is pushing the lever into the wrong direction for the big global challenges we face. Change will only happen bottom-up, because the top of the hierarchy is ruled by money, therefore change still starts at an individual level.

"Never doubt that a small group of thoughtful, committed citizens can change the world: indeed, it's the only thing that ever has." - Margaret Mead, anthropologist, recipient of the Planetary Citizen of the Year Award in 1978.

There have been many instances in history where individuals have acted with integrity and determination to make a big societal change, for example Mahatma Ghandi, Rosa Parks, Martin Luther King, Anne Frank, and many more. Let us take them as inspiration to continue to strive for the changes in the structure of a system that we deem necessary for everyone's well-being. And we can acknowledge at this level that the changes start to become really hard, for the individual as well as the group. As a reminder, that is also an indicator that they will have a big impact on the overall system. As community, we can take a pledge for changing the structure of reward systems, and thereby change the system incentives. 
Steve Easterbrook ${ }^{3}$ proposed in a conversation to limit ourselves to ten publications in a lifetime, for example, so that way we would think really well about what is worth publishing. I added this as a reflection question for myself: Would the paper at hand make the list? I believe it is a very important conversation to have.

LP 3 Goals of the system: Currently, the goals of the work world seem to be to successfully compete over resources - recognition in one's career, market share, a customer segment, and so on. Maybe we are after the wrong goals though. Leaving a legacy is not necessarily accomplished in number of sales or publications. As author Maya Angelou phrased it "Your legacy is every life you touch." So instead of trying to get a bigger piece of the cake, can we turn towards essentialism [11]? Take the time to figure out what is truly important to us and then focus on that instead? In the study on regrets of the dying, there were many who wished they had spent more time with friends and family, and even more that they had lived a life true to their own values instead of following what other people wanted them to do [23]. I question my academic contributions by the impact they will have on individuals as well as the community. There are quite a few publications I have written that do not pass this test post mortem. I believe this one does, as well as my research area of the impact of mindfulness practices on computer workers as we embed the our values into the systems we create. Here is the chance to question the goals of the system and to decide to do something to change them - the more of use dare to take steps in this direction, the more people will find their courage to do the same, and that is how I as an individual and we as community can have an impact on societal goals.

LP 2 Mindset: Our society currently seems to predominantly be based on a mindset of scarcity - that there are not enough resources for everyone, therefore we have to compete to survive. And it is hard to believe in the face of many people suffering around the world that this may not be true. Certainly, true is that the resources are very unevenly distributed and that causes many problems. What if we could shift our individual mindset to one of abundance, of trusting that we are all part of a larger organism and that there are sufficient resources to take care of everyone. It also helps to put in perspective what our actual needs are, that a hunger for more in one area does not necessarily signify that we need more of that, but our mind may tell us that. The simplest example I find for that is the tendency to eat too much under stress. For many people, this can be perceived even if they have a perfectly normal weight, but when we are under stress, we tend to overeat to fulfill a need for more energy (which would be helped by relaxing, not necessarily more food), or when we feel lonely and disconnected, we tend to overeat to fulfill a need for touch and to numb ourselves ${ }^{4}$. For myself, this example has been a struggle since I was an early teenager, and I need to watch the reasons for my eating to this day and ask whether I am hungry or whether I am trying to fill a different need. The same applies for any type of consumption, shopping or media. In the pandemic, we saw it in a disproportionate run on groceries and toilet paper, in an expression of anxiety about scarcity, while other parts of the community came together and created beautiful

\footnotetext{
${ }^{3}$ https://www.easterbrook.ca/steve/

${ }^{4}$ https://www.theschooloflife.com/thebookoflife/why-we-eat-too-much/
}

examples of communitas in terms of caring for elderly neighbors and supporting healthcare workers.

LP 1 Power to transcend paradigms: Welcome to the summit - we have arrived at the most abstract leverage point. It is to acknowledge that no paradigm that we operate under may be valid forever. We try our best when we change mindsets at paradigms at a given point in time, and that always happens in evaluation of the context at that time, which may change significantly thereafter. Eventually, another change of paradigm becomes necessary. It seems the only thing we know for sure is that everything changes and that nothing is sure. The question becomes whether we can be less attached to our perspectives and, as a result, experience more piece of mind. Can we stay consciously aware in the face of change happening? Can we trust and be okay with the uncertainty of life? Especially the pandemic has us struggle with many of our assumptions about life being challenged. I find myself grieving some previously cherished daily activities and pleasures, while taking the time to turn inward, acknowledging what is really important to me, and trusting in that I can be OK whatever happens. I choose my internal experience and how I respond to changing circumstances. They may not be pleasant, my inner experience my not be pleasant, and I choose the response to change. In other areas of science, this is called letting go of attachment [20]. For communitas, the power to transcend paradigms may be the ultimate expression of resilience.

\section{DISCUSSION}

In this section, I discuss the relevance, implementation dangers, deep work zones, the idea of simplification and less is more, what all of this could mean on an institutional level, and neurobiological impacts.

Relevance: Is all of this a vain reflection on my habits and how the world would be better if everyone did this? I thought hard about how to phrase this contribution, and instead of only quoting books about how to manage our time better. I thought it was the more honest approach to report back on the things that I have actually tried and that work well to the extent that I manage to apply them. And the point is to show how the leverage points can support us in understanding the system dynamics of our societal systems better, so we can push the levers into the direction that actually helps as opposed to aggravating the situation.

Implementation: Is there a danger of a rebound effect and pushing the lever in the wrong direction? Does the load first increase (until one "gets the hang of it") before it can get lower? The answer to that one is twofold: First, do not try to change everything at the same time, because that is for sure cognitively overwhelming and leads to the experience of failure. Second, make it the easier choice. How can I make it easier to do the 'good thing' and default to that, instead of going back to what I previously did?

Deep Work Zone: In order to facilitate deep work zone [14], we need to have some time without interruption. That said, the overall book's premise points towards a very specific value contribution that is too narrow for me ("stay competitive in a globally competitive information economy" [14]) as there seems no space to care for others in a community sense if it does not contribute to 
the competitive success metrics. Mark et al.'s [10] data suggests that people compensate for interruptions by working faster, but this comes at a price: experiencing more stress, higher frustration, time pressure and effort. Schaule et al. [18] confirmed this by using wearables on office workers to detect and compute cognitive load. A couple of guidelines that have worked for me are to close tabs, windows and applications unless needed in the immediate task at hand; writing physically on a piece of paper to slow down thoughts because of the limited writing speed and to maybe helps connect the dots better; to play brain wave music and drink lots of waterwhat does your deep work zone look like?

Less is more: We all need to get things done, and we all have a desire to communicate with each other. At the same time there is an understanding that if we are experiencing cognitive overload, maybe part of the answer lies in reducing - input as well as output. Referring to the examples above, the culture of excessive information in emails and various other digital communication channels would be one of them, and my results confirm Widdicks et al. [24, 25] in their effectiveness of decreasing internet use to improve energy levels and perceived productivity. So, when is that applicable and when not - a balance in between communication and silence. I like a quote by author Bernard Meltzer to help me decide: 'Before you speak ask yourself if what you are going to say is true, is kind, is necessary, is helpful. If the answer is no, maybe what you are about to say should be left unsaid.' However, as silent monk and Yogi Hari Das Baba already pointed out, 'the mind can't be stopped by shutting the mouth' 5 , and it takes willpower and mental training occur in holding up silence. Sensory deprivation or fasting sensory input have proven benefits in many ancient disciplines and are experiencing a renaissance in Silicon Valley and other high-paced high-tech business areas where people burn out a lot.

Simplify: Downsizing and simplification have been discussed in our lifestyle by movements like minimalism [13] and essentialism [11] and can range from eating simply (e.g. a plant-based diet) to dressing simply (designers all around the world tend to dress in black only). ${ }^{6}$ Slow science ${ }^{7}$ helped me with my commitment to fewer publications, as also referenced by Fullerton quoting the author Salinger on his experience of 'the lightness of not publishing' [4]), so that I can focus on only the ones that matter according to my new perception of likely practical impact. Finally, shorter working times have been tested in Göteborg, Sweden, with the only group of workers that is even more likely to experience depression by their work - nursing. They reduced to a 6-hour workday in 2015 and found happier employees, less sick days, and improved patient care [9]. Science has also proven sufficiently that the cognitive abilities of workers doing think-work goes down significantly after about six hours of work, so why should we continue to push past the boundary into declining returns on investment?

Institutional level: What does this mean on an institutional level? Does this help to increase awareness? Is awareness sufficient? No,

\footnotetext{
${ }^{5}$ Hari Das Baba, silent monk and yogi, see www.babaharidass.org

${ }^{6}$ As a yoga teacher I get away with wearing yoga pants any given day, and it makes the choice in the morning of what to wear so much simpler.

${ }^{7}$ http://www.slowscience.org
}

it is not, but it is a precondition for change to be able to happen. Assuming I have individuals who decide to make changes, at what leverage point is it necessary to come to a group consensus for implementing change as opposed to an individual deciding for themselves? How do I understand where the boundary is? For example, I can decide as an individual to only answer emails twice daily, and there are good reasons for doing so, like uninterrupted deep work time and that email is an asynchronous communication medium in the first place. But what if my company has an 'always online' policy? Can I decide for myself to go rogue and simply await consequences, or is it better to have a larger conversation on whether the 'always online' policy is actually more detrimental to everyone's productivity and quality of work than it is beneficial for resolving issues quickly? If I am the only person applying the change, I might see a benefit for myself, but the compound benefit of a team or organization would be much larger. Options would be to offer preventative measures and training focus; like fitness, yoga, breathwork, meditation, nutrition, wellness, or a running group.

Integration with neuroscientific theory: In order to learn something that has an effect on behavior, not only the mind, but also both the body and the emotions have to be involved. Only if there is enthusiasm, it will be possible to overwrite previous patterns and habits with positively connotated new behavior [5]. Integrating the body means moving the body, another critical element in our computer-work dominated offices, and if we manage to tie in some of the targeted behavior changes with physical movement, we can make a big step forward in succeeding to change successfully in the long run.

\section{CONCLUSION}

This paper used the lens of leverage points to look at ways of how to lower cognitive load for computer work and increase the flow and resilience for individuals as well as groups. This discourse was presented specifically in the context of an explorative qualitative study of observations and reflections of mine over the course of a decade.

How do humans transition towards more individual sustainability and resilience in the context of computer work? According to Smith and Raven [22, p. 1025], "Sustainability transitions research emphasises the role of niches as a source for path-breaking innovation. A defining characteristic of these niches is that they afford temporary 'protective space' for the configuration and development of such innovations." Such protective spaces are mentioned in the discussion, and efforts have been undertaken at many institutions, but without adjusting the feedback loops and rules around the existing system, simply adding on such an element of a protective space as a buffer does not have sufficient impact to make a change of the necessary extent.

Most importantly, this paper points out that the individual can have an impact on the system (via the leverage points) in the way they contribute to communitas, influence group energy, and create momentum towards change. And this momentum is much needed in the face of current uncertainty.

How do you best connect with flow and communitas? 


\section{ACKNOWLEDGMENTS}

I thank Julia Roelofsen for thoughtful input on an early draft of this paper. Furthermore, I thank Regina Hebig and Salome Honest Maro for insightful conversations on the topic. Finally, I thank Elina Eriksson, Douglas Schuler, and Lisa Nathan for valuable and thoughtful feedback in their reviews.

\section{REFERENCES}

[1] David J Abson, Joern Fischer, Julia Leventon, Jens Newig, Thomas Schomerus, Ulli Vilsmaier, Henrik von Wehrden, Paivi Abernethy, Christopher D Ives, Nicolas W Jager, et al. 2017. Leverage points for sustainability transformation. Ambio 46, 1 (2017), 30-39.

[2] Mihaly Csikszentmihalyi, Sami Abuhamdeh, and Jeanne Nakamura. 2014. Flow. In Flow and the foundations of positive psychology. Springer, 227-238.

[3] Jay W Forrester. 1971. World Dynamics,(1973.

[4] Ben Fullerton. 2010. Designing for solitude. interactions 17, 6 (2010), 6-9.

[5] Gerald Hüther. 2016. Mit Freude lernen-ein Leben lang: Weshalb wir ein neues Verständnis vom Lernen brauchen. Sieben Thesen zu einem erweiterten Lernbegriff und eine Auswahl von Beiträgen zur Untermauerung. Vandenhoeck \& Ruprecht.

[6] WonYang Kang, Won-Ju Park, Keun-Ho Jang, Hyeong-Min Lim, Ji-Sung Ann, Seung-hyeon Cho, and Jai-Dong Moon. 2016. Comparison of anxiety and depression status between office and manufacturing job employees in a large manufacturing company: a cross sectional study. Annals of occupational and environmental medicine 28, 1 (2016), 47.

[7] Taeshik Kim, Mo-Yeol Kang, Min-sang Yoo, Dongwook Lee, and Yun-Chul Hong. 2016. Computer use at work is associated with self-reported depressive and anxiety disorder. Annals of occupational and environmental medicine 28, 1 (2016), 57.

[8] Steven Kotler and Jamie Wheal. 2017. Stealing fire: How Silicon Valley, the Navy SEALs, and maverick scientists are revolutionizing the way we live and work. HarperCollins.

[9] Bengt Lorentzon. 2019. Longitudinal, experimental evaluation of reduced weekly working hours for assistant nurses. (2019).
[10] Gloria Mark, Daniela Gudith, and Ulrich Klocke. 2008. The cost of interrupted work: more speed and stress. In Proceedings of the SIGCHI conference on Human Factors in Computing Systems. 107-110.

[11] Greg McKeown. 2014. Essentialism: The disciplined pursuit of less. Currency.

[12] Donella Meadows. 1999. Leverage points. Places to Intervene in a System (1999).

[13] Joshua Fields Millburn and Ryan Nicodemus. 2015. Minimalism: Live a meaningful life. Asymmetrical Press.

[14] Cal Newport. 2016. Deep work: Rules for focused success in a distracted world. Hachette UK.

[15] Daniel Pargman and Barath Raghavan. 2014. Rethinking sustainability in computing: From buzzword to non-negotiable limits. In Proceedings of the 8th Nordic Conference on Human-Computer Interaction: Fun, Fast, Foundational. 638-647.

[16] Birgit Penzenstadler. 2020. Where attention goes, energy flows - enhancing individual sustainability in software engineering. In Proceedings of the Intl. Conf. on ICT for Sustainability.

[17] Birgit Penzenstadler, Leticia Duboc, Colin C Venters, Stefanie Betz, Norbert Seyff, Krzsztof Wnuk, Ruzanna Chitchyan, Steve M Easterbrook, and Christoph Becker. 2018. Software engineering for sustainability: Find the leverage points! IEEE Software 35, 4 (2018), 22-33.

[18] Florian Schaule, Jan Ole Johanssen, Bernd Bruegge, and Vivian Loftness. 2018. Employing consumer wearables to detect office workers' cognitive load for interruption management. Proceedings of the ACM on Interactive, Mobile, Wearable and Ubiquitous Technologies 2, 1 (2018), 1-20.

[19] Phoebe Sengers. 2011. What I learned on Change Islands: reflections on IT and pace of life. Interactions 18, 2 (2011), 40-48.

[20] Michael Singer. 2007. The untethered soul: The journey beyond yourself. New Harbinger Publications.

[21] Derek Sivers. 2009. No 'Yes.'Either 'HELL YEAH!'or 'No'. Sivers. Published August 26 (2009).

[22] Adrian Smith and Rob Raven. 2012. What is protective space? Reconsidering niches in transitions to sustainability. Research policy 41, 6 (2012), 1025-1036.

[23] Susie Steiner. 2012. Top five regrets of the dying. the Guardian (2012).

[24] Kelly Widdicks and Daniel Pargman. 2019. Breaking the Cornucopian Paradigm: Towards Moderate Internet Use in Everyday Life. In Proceedings of the Fifth Workshop on Computing within Limits. 1-8.

[25] Kelly Victoria Widdicks, Tina Ringenson, Daniel Pargman, Vishnupriya Kuppusamy, and Patricia Lago. 2018. Undesigning the Internet: An exploratory study of reducing everyday Internet connectivity. (2018). 